\title{
SPECTRAL ASYMPTOTICS FOR FOURTH ORDER DIFFERENTIAL OPERATOR WITH TWO TURNING POINTS
}

\author{
L.G. VALIULLINA, Kh.K. ISHKIN, R.I. MARVANOV
}

\begin{abstract}
The paper is devoted to studying the asymptotics of the spectrum of a self-adjoint operator $T$ generated by a fourth-order differential expression in the space $L^{2}(0,+\infty)$ under the assumption that the coefficients of the expression have a power growth at infinity such that: a) the deficiency index of the corresponding minimal operator is $(2,2)$, b) for sufficiently large positive values of a spectral parameter, the differential equation $T y=\lambda y$ has two turning points: a finite one and $+\infty, \mathrm{c}$ ) the roots of the characteristic equation grow not with the same rate. The latter assumption leads one to significant difficulties in studying the asymptotics of the counting function for the spectrum by the traditional Carleman-Kostyuchenko method based on estimates of the resolvent far from the spectrum and Tauberian theorems. Curiously enough, the method of reference equations used to solve the more subtle problem of finding asymptotic expansions of the eigenvalues themselves, and therefore more sensitive (compared to the Carleman-Kostyuchenko method) to the behavior of the coefficients in the differential expression is more effective in the considered situation: imposing some constraints on coefficients such as smoothness and regular growth at infinity, we obtain an asymptotic equation for the spectrum of the operator $T$. This equation allows one to write out the first few terms of the asymptotic expansion for the eigenvalues of the operator $T$ in the case when the coefficients have a power growth. We also note that so far the method of reference equations has been used only in the case of the presence of the only turning point.
\end{abstract}

Keywords: differential operators, spectral asymptotics, turning point, singular numbers

Mathematics Subject Classification: 34B40, 34L20, 34L40, 47E05

\section{INTRODUCTION}

Let $T$ be a lower semi-bounded self-adjoint operator acting in some infinite-dimensional separable Hilbert space with a discrete spectrum. The latter means that the spectrum consists of countably many eigenvalues of finite multiplicity and possess the unique accumulation point $+\infty$. Let $\left\{\lambda_{n}\right\}_{n=1}^{\infty}$ be the eigenvalues of the operator $T$ taken in the ascending order counting multiplicities. The asymptotic behavior of the sequence $\left\{\lambda_{n}\right\}_{n=1}^{\infty}$ can be written in terms of the eigenvalues or in terms of the counting function of the spectrum $N(\lambda)=\sum_{\lambda_{n}<\lambda} 1$ :

$$
\begin{aligned}
\lambda_{n} & \sim f(n), & & n \rightarrow+\infty, \\
N(\lambda) & \sim g(\lambda), & & \lambda \rightarrow+\infty,
\end{aligned}
$$

where $f, g$ are some increasing mutually inverse functions defined in some neighbourhood of the point $+\infty$. Formulae (1) and (2) are not always equivalent. For instance, if $f(\lambda)=C e^{\lambda}$, where $C$ is a positive constant, then formula (1) implies (2). By formula (2) is equivalent

L.G. Valiullina, Kh.K. Ishkin, R.I. Marvanov, Spectral asymptotics for fourth Order DifFERENTIAL OPERATOR WITH TWO TURNING POINTS.

(c) Valiullina L.G., IshKin Kh.K., Marvanov R.I. 2018.

The research is financially supported by a grant of Russian Science Foundation (project no. 18-11-00002).

Submitted June 7, 2018. 
to $N(\lambda) \sim \ln \lambda, \lambda \rightarrow+\infty$, which obviously does not yield (1). It is easy to check that as $g(\lambda)=C e^{\lambda}$, formula (2) is stronger than (1).

The considered examples show that the non-equivalence of formulae (1) and (2) is not only since the function $N(\lambda)$ is defined up to $O(1)$ but because of how "well" the behavior of the functions $f$ and $g$ at infinity is. The condition of the "well" behavior is well-known [1]: if

$$
\lim _{\delta \rightarrow 1+0} \limsup _{x \rightarrow+\infty} \frac{f(\delta x)}{f(x)}=1,
$$

then for each increasing sequence $\left\{\lambda_{n}\right\}$ with asymptotics (2), estimate (1) is true as well. The same statement is true for the implication $(1) \Rightarrow(2)$. The functions obeying condition (3) are called pseudo regularly varying functions (PRV-functions). Because of numerous applications, especially in probability theory, PRV-functions were studied rather well, see [2] and the references therein. We note that PRV-functions are natural generalizations of RV-class of regularly varying functions introduced by Karamata in 1903 in its basic work [3]. The results by Karamata together with further developments and generalizations turned out to be exceptionally fruitful for various fields in mathematics, see [4,5].

Being applied, condition (3) is not always effective. Suppose that for some operator we succeed to obtain estimate (2) but we need to find a more precise asymptotics

$$
\lambda_{n} \sim f(n)+O\left(\alpha_{n}\right), \quad n \rightarrow+\infty,
$$

where sequence $\left\{\alpha_{n}\right\}$ decays with a prescribed rate. It is clear that even if the function $g$ satisfies condition (3), formula (4) should not follow (2). Thus, under condition (3), formula (4) can be stronger than formula (2).

A more essential difference is between the methods of obtaining estimates (4) and (2). One of the main methods for studying asymptotics $N(\lambda)$ going back to work [6] by Carleman is based on estimating the resolvent $(T-\lambda)^{-1}$ or some function of the resolvent for large $\lambda$ far from the spectrum of $T$ followed by applying Tauberian theorems, see, for instance, [7]. We note that Tauberian method can be successfully applied also to non-self-adjoint operators [8], [9]. But once we want to find several first terms in asymptotics expansion (4), the Tauberian technique is no longer applicable. The reason is that we have to descend to the spectrum and to study the asymptotics of solutions to the equation $T y=\lambda y$ as $\lambda$ goes to infinity along a set containing the spectrum of the operator $T$. In the case, when $T$ is a singular ordinary differential operator [10], the latter circumstance usually gives rise to turning points [11, Ch. III, Sect. 1], which complicate essentially the problem on finding asymptotic expansions for solutions to the equation $T y=\lambda y$. This is why, at least for ordinary differential operators, the problem on finding expansion (4) is often more complicated than a similar problem for $N(\lambda)^{1}$. In this context, an operator, for which formula (4) can be obtained easier than (2), should be regarded as a curious incident.

The paper is devoted to obtaining formula (4) for one of such operators. This operator, denoted by $T$, acts in the space $L^{2}(0,+\infty)$ by the rule:

$$
T y=\mathcal{L}(y):=y^{(4)}-2\left(p(x) y^{\prime}\right)^{\prime}+q(x) y
$$

on the functions

$$
\begin{aligned}
& D(T)=\left\{y \in L^{2}(0,+\infty): y^{[k]} \in A C[0,+\infty)(k=\overline{0,3})\right. \\
&\left.\mathcal{L}(y) \in L^{2}(0,+\infty), y(0)=y^{\prime \prime}(0)=0, \lim _{x \rightarrow+\infty}[y, y](x)=0\right\} .
\end{aligned}
$$

\footnotetext{
${ }^{1}$ For some operators, for instance, for partial differential operators, the leading term in expansion (1) can be found only from (2) by inverting the function $g$.
} 
Here

$$
[y, z]=\sum_{k=1}^{2}\left(y^{[k-1]} \bar{z}^{[2-k]}-y^{[2-k]} \bar{z}^{[k-1]}\right),
$$

$y^{[k]}$ is the $k$ th quasi-derivative $\left[10\right.$, Sect. 15] of a function $y$ : $y^{[k]}=y^{(k)}$ as $k=\overline{0,2}$ and $y^{[3]}=2 p y^{\prime}-y^{\prime \prime \prime}$. The symbol $A C[0,+\infty)$ stands for the set of functions absolutely continuous on each segment $[0, b], b>0$. Hereinafter we assume that the functions $p$ and $q$ are real and summable on each interval $(0, b), b>0$. Under such assumptions, $T$ is a closed symmetric operator with the deficiency index $(n, n)$, where $0 \leqslant n \leqslant 2$, see [10, Sect. 17, Subsect. 5]. If $T$ is self-adjoint, that is, $n=0$, and for some $a>0$ the functions $p$ and $q$ are non-negative almost everywhere in $(a, \infty)$, then it follows from the proof of Lemma 2 in work [12] that $T$ coincides with the operator associated with a lower-bounded closed quadratic form

$$
\begin{aligned}
l[y] & =\int_{0}^{\infty}\left(\left|y^{\prime \prime}\right|^{2}+2 p\left|y^{\prime}\right|^{2}+q|y|^{2}\right) d t \\
D(l) & =\left\{y \in L^{2}(0, \infty): \sqrt{q} y, \sqrt{p} y^{\prime} \in L^{2}(a, \infty), y^{\prime \prime} \in L^{2}(0, \infty), y(0)=0\right\},
\end{aligned}
$$

and hence, it is lower semi-bounded. If in addition we assume that

$$
q(x) \rightarrow+\infty, x \rightarrow \infty,
$$

then by the minimax principle [13, Ch. XIII, Sect. 1], the operator $T$ has a discrete spectrum.

As it has been mentioned above, to obtain asymptotic estimates (1) or (2), it is important to know the behavior of fundamental system of solutions (FSS) to the equation

$$
y^{(4)}-2\left(p(x) y^{\prime}\right)^{\prime}+q(x) y=\lambda y
$$

for large $x>0$ and $\lambda>0$, respectively, for $\lambda<0$. The behavior of FSS depends essentially on the behavior of characteristic roots $\mu_{i}(x, \lambda),(i=\overline{1,4})$, of the equation

$$
\mu^{4}-2 p \mu^{2}+q-\lambda=0 \text {. }
$$

We have:

$$
\mu_{1,2}= \pm \sqrt{\nu_{1}}, \quad \mu_{3,4}= \pm \sqrt{\nu_{2}}
$$

where $\nu_{1,2}=p \pm \sqrt{D}, D=p^{2}+\lambda-q$. Hereinafter the branch of the root $\sqrt{z}$ is chosen so that $\sqrt{z}>0$ as $z>0$. Thus, if $q$ satisfies (6) and

$$
p^{2}(x)=o(q(x)), \quad x \rightarrow \infty,
$$

there exist positive constants $A, B$ and $C$ such that as $x>a, \lambda<-C, k, j=\overline{1,4}$, we have

$$
B \leqslant\left|\frac{\mu_{k}(x, \lambda)}{\mu_{j}(x, \lambda}\right| \leqslant A .
$$

Conditions (10) play important role while deriving estimate (2) for quasi-differential operator of arbitrary order [7, Ch. VIII]. As this condition fails, the asymptotic structure of FSS for equation (7) can become much more complicated, see [14] and the references therein. First, this complexity is due to the fact that the characteristic roots grow not at the same rate at infinite, which is a degenerate case. For instance, if $p(x) \rightarrow+\infty, x \rightarrow \infty$, and

$$
q(x)=o\left(p^{2}(x)\right), \quad x \rightarrow \infty,
$$

then

$$
\mu_{j+2}(x, \lambda)=o\left(\mu_{j}(x, \lambda)\right) \quad(j=1,2), \quad x \rightarrow+\infty .
$$

Second, part of these roots can glue into a multiple root at some points called turning points. As it has been mentioned above, the presence of the latter cause many troubles in studying the asymptotics of FSS, see [11], [15] and further references. Moreover, if

$$
q(x)=x^{\alpha}, \quad p(x)=\sqrt{x^{2 \beta}+x^{\gamma} \cos x^{\delta}+x^{\alpha}}, \quad 0<\alpha, \gamma<2 \beta, \quad \delta>0,
$$


then by formulae (8) we see that as $\lambda=-r, r \gg 1$, subject to the sign of $\delta+\gamma-2 \beta$, equation (7) has either finite or infinite number of turning points determined by the equation $x^{2 \beta}+x^{\gamma} \cos x^{\delta}=r$. In the vicinity of each turning point, the standard asymptotic estimates (WKB-estimates for second order equations and their analogues for higher order equations and systems [11]) do not work.

This is why under condition (11) and for large negative $\lambda$ we have to deal with troubles of both types. Nevertheless, Carleman-Kostyuchenko method for obtaining formula (2) can be also applied in this situation, we just should go to the complex $\lambda$-plane (see [14 and also [7]).

However, in the degenerate case and of course with a more detailed information on behavior of the functions $p$ and $q$ one can succeed to obtain formula (4) under imposed conditions for $p$ and $q$ and this formula is stronger than (2). At that, the troubles cased by the presence of the turning points turn out to be less than in problem on finding formula (2)!

There different methods thank to which one can overcome problems related with the turning points and to obtain formula (2) for operators $T$. One of them, see, for instance, [14], [16], is the aforementioned going to the complex $\lambda$-plane, namely, the studying of the asymptotics of the Green function of the operator $T$ for large $\lambda$ in some non-real ray leaving the origin and the applying of some Tauberian theorem [8], [9], [17]. Another method coming back to Langer's work [18] allows one to obtain an approximate solution to equation (7) suitable both at the turning point and far from it. This method is effective in self-adjoint and non-self-adjoint spectral problems [15], [19], [20]. Exactly thanks to Langer's method, one succeeds to obtain formula (4) with the estimate

$$
\alpha_{n}=n^{-m}, \quad m=\mathrm{const}>0,
$$

in degenerate case (11), when the coefficients $p$ and $q$ grow at a power rate and satisfy some additional smoothness conditions and conditions of regular growth at infinity, cf. Theorem 2 . We stress once again: on one hand, estimates (4) and (13) involve much more information about the spectrum of the asymptotics of the operator $T$ in comparison with estimate (2) and on the other hand, Langer's method can be helpless for obtaining estimate (2) in some case, as in example (12)).

\section{Formulation of MAin Results}

We impose the following restrictions on real functions $p$ and $q$ :

1) There exists $x_{0}>0$ such that the functions $p$ and $q$ are summable on $\left(0, x_{0}\right)$.

2) As $x \geqslant x_{0}$, where $x_{0} \geqslant 0$ is a constant, the functions $p$ and $q$ possess absolutely continuous derivatives satisfying the inequalities:

$$
a_{1} x^{\alpha-1} \leqslant q^{\prime}(x) \leqslant A_{1} x^{\alpha-1}, \quad b_{1} x^{\beta-1} \leqslant p^{\prime}(x) \leqslant B_{1} x^{\beta-1},
$$

where $a_{1}, A_{1}, b_{1}, B_{1}, \alpha, \beta$ are positive constants, and

$$
\alpha<2 \beta
$$

the second derivatives of the functions $p$ and $q$ are sign-definite almost everywhere.

Remark 1. It follows from inequalities (14) that as $x \geqslant x_{1}\left(x_{1} \geqslant x_{0}\right)$,

$$
a x^{\alpha} \leqslant q(x) \leqslant A x^{\alpha}, \quad b x^{\beta} \leqslant p(x) \leqslant B x^{\beta},
$$

where $a, A, b, B$ are positive constants. Therefore, [10, Sect. 24, Thm. 2], the spectrum of each self-adjoint extension of the minimal operator generated by expression (5) is discrete.

In what follows, under additional restrictions for the functions $p$ and $q$ we obtain a double asymptotics for solutions to the equation $\mathcal{L}(y)=\lambda y$, see [11, Ch. II, Sect. 7]. In particular, this implies that the deficiency indices of the minimal operator $T_{0}$ generated by the differential 
expression $\mathcal{L} y$ in $L^{2}(0,+\infty)$ (see [10, Ch. V, Sect. 17]) is equal to $(2,2)$. The latter fact yields the self-adjointness of the operator $T$.

By relations (8) and inequalities (15), (16) we see that for each $\lambda \gg 1$, equation (7) possesses the unique turning point $a_{\lambda}$ determined by the condition $q\left(a_{\lambda}\right)=\lambda$. At this point, there merge the roots $\mu_{3,4}$ coinciding with the characteristic root for the Sturm-Liouville equation

$$
-y^{\prime \prime}+\frac{q-\lambda}{p+\sqrt{p^{2}+\lambda-q}} y=0 .
$$

In work [21], under Conditions 1), 2) and $0<\beta<\alpha+2$, the asymptotics of FSS to equation (7) was found for large $\lambda>0$; this asymptotics was uniform in $x \geqslant 0$. Employing this asymptotics, there was obtain an asymptotic equation for the spectrum allowing one to obtain several first terms in expansion (4), (13) in the case $q(x)=x^{\alpha}, p(x)=x^{\beta}$.

While constructing asymptotics of FSS, it turned out that for large $\lambda$, two solutions in FSS can be approximated uniformly in $x \geqslant 0$ by solutions of equation (17). In the case $\beta \geqslant \alpha+2$ such approximation became inappropriate in a neighbourhood of the infinity; this neighbourhood depended on $\lambda>0$. That related with the fact that as $\beta \geqslant \alpha+2$, the point $x=+\infty$ is also a turning point and this is to construct the asymptotics of solutions to equation (17) at infinity one had to choose another reference equation. While choosing such equation, we shall need more detailed in comparison with Condition 2) information on behavior of the functions $p$ and $q$ at infinity. Namely, we assume that the function $p$ satisfies the following condition:

3) $p^{(k)}(x)=\left(x^{\beta}\right)^{(k)}+O\left(x^{\beta-k-\varepsilon}\right)$ on $\left[x_{0}, \infty\right) k=0,1,2, \varepsilon>0$.

In the case $\beta=\alpha+2$ we impose one more condition:

4) $q(x)=x^{\alpha}+O\left(x^{\alpha-\sigma}\right), \sigma>0$, on $\left[x_{0},+\infty\right)$.

It is obvious that the functions of form

$$
p(x)=x^{\beta}+R(x), \quad q(x)=x^{\alpha}+V(x),
$$

with $R, V \in C_{0}[0,+\infty)$ satisfy Conditions 1$\left.)-4\right)$.

Theorem 1. Assume that as $\beta \geqslant \alpha+2$, Conditions 1)-3) hold and as $\beta=\alpha+2$, Condition 4) is satisfied as well. Then the eigenvalues of the operator $T$ with sufficiently large indices are determined by the equation

$$
\sin \Phi(\lambda)+K(\lambda) \cos \Phi(\lambda)+O\left(\lambda^{-\delta}\right)=0
$$

where

$$
\begin{aligned}
& \Phi(\lambda)=\sqrt{\lambda} \int_{0}^{\infty}\left(p+\sqrt{p^{2}+\lambda}\right)^{-\frac{1}{2}} d t+\frac{\pi}{4}-\frac{\pi}{2(\beta-2)} \sqrt{(\beta-1)^{2}+2 C}, \\
& C= \begin{cases}0 & \text { as } \beta>2+\alpha, \\
1 & \text { as } \quad \beta=2+\alpha,\end{cases} \\
& K(\lambda)=-\frac{5}{72}\left(\Phi(\lambda)-\frac{\pi}{4}\right)^{-1}+\frac{1}{2} \int_{0}^{a_{\lambda}}\left|\nu_{2}\right|^{-\frac{1}{2}}\left(b^{2}+b^{\prime}-K(t, \lambda)+\frac{D^{\prime} \nu_{2}^{\prime} \nu_{2}}{8 D^{2}}\right) d t \\
& b=\frac{p^{\prime}}{2 \sqrt{D}}, \quad \nu_{2}=p-\sqrt{D}, \quad D=p^{2}+\lambda-q, \\
& \delta= \begin{cases}\min \left\{\frac{1}{2}, \frac{2(\beta-\alpha-2)}{\beta-2}, \frac{2 \varepsilon}{\beta-2}\right\} \quad \text { as } \beta>\alpha+2, \\
\min \left\{\frac{1}{2}, \frac{2 \sigma}{\beta-2}, \frac{3 \varepsilon}{\beta-2}\right\} \quad \text { as } \beta=\alpha+2 .\end{cases}
\end{aligned}
$$


If the functions $p$ and $q$ are of form (18), then

$$
\delta= \begin{cases}\min \left\{\frac{1}{2}+\frac{1}{\beta}, \frac{2(\beta-\alpha-2)}{\beta-2}\right\} & \text { as } \beta>\alpha+2 \\ \frac{1}{2}+\frac{1}{\beta} & \text { as } \beta=\alpha+2 .\end{cases}
$$

Theorem 2. Assume that the functions $p$ and $q$ are of form (18). Then for $2+\alpha<\beta<$ $2+2 \alpha$ the spectrum of the operator $T$ has the asymptotics:

$$
\lambda_{k}=m_{k}^{\frac{4 \beta}{\beta+2}}+\frac{4 \beta}{\beta+2} C_{0}^{-1}\left\{C_{1} m_{k}^{\frac{2(\beta-1)}{\beta+2}}-C_{2} m_{k}^{\frac{2(\beta-2)}{\beta+2}}+C_{3} m_{k}^{\frac{\left((\beta-2)^{2}+2 \alpha \beta\right)}{\beta^{2}-4}}\right\}+O\left(k^{-m}\right),
$$

where

$$
\begin{aligned}
& m_{k}=C_{0}^{-1} \pi\left(k+\frac{\beta}{4(\beta-2)}\right), \\
& C_{0}=\int_{0}^{\infty}\left(x^{\beta}+\sqrt{x^{2 \beta}+1}\right)^{-\frac{1}{2}} d x, \quad C_{1}=\frac{1}{2} \int_{0}^{\infty} R(x) d x, \\
& C_{2}=\frac{\beta}{8} \int_{0}^{\infty}\left[\widetilde{\nu_{2}} \frac{1}{2} \widetilde{D}^{-\frac{1}{2}} t^{\beta-2}\left(-2 \beta \widetilde{D}^{-1}+\beta \widetilde{D}^{-2}-\frac{3}{4} \beta t^{\beta} \widetilde{D}^{-\frac{1}{2}}+1\right)+\right. \\
& \left.+\frac{3 \beta-4}{(\beta-2)^{2}} \widetilde{Q}^{-2} \widetilde{\nu}^{-\frac{1}{2}}\right] d t-\frac{(3 \beta-4) \beta}{8(\beta-2)^{2}} C_{0}^{-1} \\
& \widetilde{\nu}=t^{\beta}+\sqrt{\widetilde{D}}, \quad \widetilde{D}=t^{2 \beta}+1, \quad \widetilde{Q}=\int_{t}^{\infty} \widetilde{\nu}^{-\frac{1}{2}} d t, \quad \gamma=\frac{\beta-1}{\beta-2}, \\
& C_{3}=\frac{\pi}{2}\left(\frac{\beta-2}{\sqrt{2}}\right)^{-\frac{2 \alpha}{\beta-2}} \int_{0}^{\infty} t^{-\frac{2 \alpha}{\beta-2}+1} J_{\gamma}^{2}(t) d t, \quad J_{\gamma} \quad \text { is the Bessel function of first kind, } \\
& m=\min \left\{\frac{1}{2}+\frac{1}{\beta}, \frac{3}{4}, \frac{2(\beta-\alpha-2)}{\beta-2}, \frac{1}{2}+\frac{\beta-\alpha-2}{\beta-2}\right\}-\frac{3 \beta-2}{\beta+2} .
\end{aligned}
$$

As $\beta \geqslant 2+2 \alpha$, similar formulae hold true.

\section{Proof of Theorems 1}

3.1. Reduction of main equation to canonical form. We introduce notations. Let $\chi(x)$ be an infinitely differentiable function equalling one on $\left[0, x_{0}\right]$ and vanishing on $\left[x_{0}+1, \infty\right)$. We denote

$$
\begin{aligned}
& p_{1}(x)=p(x)(1-\chi(x)), \quad q_{1}(x)=q(x)(1-\chi(x)), \quad f(x, \lambda, \mu)=\mu^{4}-2 p_{1} \mu^{2}+q_{1}-\lambda, \\
& A_{1}=\left(\begin{array}{cccc}
0 & 1 & 0 & 0 \\
0 & 0 & 1 & 0 \\
0 & 2 p_{1} & 0 & -1 \\
q_{1}-\lambda & 0 & 0 & 0
\end{array}\right), \quad A_{2}=\chi\left(\begin{array}{cccc}
0 & 0 & 0 & 0 \\
0 & 0 & 0 & 0 \\
0 & 2 p & 0 & 0 \\
q & 0 & 0 & 0
\end{array}\right) .
\end{aligned}
$$

We let $Y=\left(y, y^{[1]}, y^{[2]}, y^{[3]}\right)^{T}$, where $y^{[k]}$ stands for the $k$ th quasi-derivative [10]. Then the equation $\mathcal{L} y=\lambda y$ is equivalent to the system of equations

$$
Y^{\prime}=\left(A_{1}+A_{2}\right) Y \text {. }
$$

We introduce the matrices

$$
\begin{aligned}
& A_{0}=\operatorname{diag}\left(A_{01}, A_{02}\right), \\
& A_{01}=\sqrt{\nu_{1}} \operatorname{diag}(1,-1), \quad A_{02}=\left(\begin{array}{cc}
0 & 1 \\
\nu_{2} & 0
\end{array}\right),
\end{aligned}
$$




$$
T=D^{-\frac{1}{4}}\left(\begin{array}{cc}
I_{2} & I_{2} \\
\Lambda_{1} & \Lambda_{2}
\end{array}\right) \operatorname{diag}\left(M W, I_{2}\right)
$$

where $I_{n}$ is the unit matrix of $n$th order,

$$
\begin{array}{ll}
\Lambda_{1}=\operatorname{diag}\left(\nu_{1},-\nu_{2}\right), & \Lambda_{2}=\operatorname{diag}\left(\nu_{2},-\nu_{1}\right), \\
W=\left(\begin{array}{cc}
1 & 1 \\
1 & -1
\end{array}\right), & M_{1}=\operatorname{diag}\left(\nu_{1}^{-\frac{1}{4}}, \nu_{1}^{\frac{1}{4}}\right), \\
B_{1}=-T^{-1} T^{\prime}, & B_{2}=T^{-1} A_{2} T .
\end{array}
$$

We let

$$
\begin{aligned}
& B_{1}=\left(\begin{array}{ll}
B_{11} & B_{12} \\
B_{21} & B_{22}
\end{array}\right), \quad X=\left(\begin{array}{ll}
X_{11} & X_{12} \\
X_{21} & X_{22}
\end{array}\right), \\
& X_{11}=-\frac{1}{2} A_{01}^{-1} B_{11}, \quad X_{22}=-\frac{p}{2 \sqrt{D}}\left(\begin{array}{ll}
0 & 0 \\
1 & 0
\end{array}\right), \\
& X_{12}=-\frac{1}{2 \sqrt{D}}\left(A_{01}\left(B_{12}+B_{12} A_{02}\right)\right. \\
& X_{21}=-\frac{1}{2 \sqrt{D}}\left(B_{21} A_{01}+A_{02} B_{21}\right) .
\end{aligned}
$$

The following relations can be checked easily:

$$
T^{-1} A T=A_{0}, \quad X A_{0}-A_{0} X=B_{1} .
$$

Then the substitution

$$
Y=T\left(I_{4}+X\right) V
$$

reduces equation 26 to the form

$$
V^{\prime}=\left(A_{0}+Z_{1}\right) V
$$

where

$$
Z_{1}=\left(I_{4}+X\right)^{-1}\left(B_{1} X-X^{\prime}+B_{2}\left(I_{4}+X\right)\right) .
$$

3.2. reference solutions. We introduce the notations:

$$
\begin{array}{ll}
\xi=\left(\frac{\beta-2}{2} \int_{x}^{\infty} \frac{d t}{\nu^{\frac{1}{2}}}\right)^{-\frac{2}{\beta-2}}, \quad \nu=p_{1}+\sqrt{p_{1}^{2}+\lambda} \\
B=\left(\xi^{\prime}\right)^{-\frac{1}{2}}, \quad Q_{1}=\int_{0}^{x} \nu_{1}^{\frac{1}{2}} d t, & Q_{2}=\sqrt{\lambda} \int_{x}^{\infty} \frac{d t}{\nu^{\frac{1}{2}}}
\end{array}
$$

We choose reference solutions as

$$
\begin{array}{ll}
V_{0}=\operatorname{diag}\left(V_{01}, V_{02}\right), & V_{01}=\operatorname{diag}\left(\exp Q_{1}, \exp \left(-Q_{1}\right)\right), \quad V_{02}=\left(\begin{array}{ll}
v_{1} & v_{2} \\
v_{1}^{\prime} & v_{2}^{\prime}
\end{array}\right), \\
v_{1}=B \xi^{\frac{1}{2}} J_{\gamma}\left(Q_{2}\right), & v_{2}=B \xi^{\frac{1}{2}} Y_{\gamma}\left(Q_{2}\right), \\
\gamma= \begin{cases}\frac{\beta-1}{\beta-2} & \text { as } \beta>\alpha+2, \\
\frac{\sqrt{(\beta-1)^{2}+2}}{\beta-2} & \text { as } \beta=\alpha+2 .\end{cases}
\end{array}
$$

Here $J_{\gamma}$ and $Y_{\gamma}$ are Bessel function of first and second kind, respectively, [22]. Then

$$
V_{0}^{\prime}=\left(A_{0}+Z_{2}\right) V_{0},
$$

where

$$
Z_{2}=\operatorname{diag}\left(0, Z_{0}\right)
$$




$$
Z_{0}=\left[\left(\frac{\beta(\beta-2)}{4}+\frac{C}{2}\right)\left(\frac{\xi^{\prime}}{\xi}\right)^{2}+\frac{B^{\prime \prime}}{B}-\frac{q_{1}}{\nu_{1}}\left(1-\frac{\lambda}{\nu\left(\sqrt{D}+\sqrt{p_{1}^{2}+\lambda}\right)}\right)\right]\left(\begin{array}{ll}
0 & 0 \\
1 & 0
\end{array}\right)
$$

and the constant $C$ is defined by formula $(21)$.

We introduce the functions

$$
\varphi=\frac{\lambda}{\nu}, \quad p_{0}=\left\{\begin{array}{lll}
\sqrt{\varphi} & \text { as } & x \leqslant b_{\lambda}, \\
\frac{1}{x} & \text { as } & x>b_{\lambda},
\end{array}\right.
$$

where $b_{\lambda}$ is the root of the equation $\sqrt{\lambda} Q_{2}\left(b_{\lambda}, \lambda\right)=1$. It is easy to see that for sufficiently large $\lambda>0$ the root $b_{\lambda}$ is determined uniquely. We also let

$$
\begin{aligned}
& P=\operatorname{diag}\left(1,1,1, p_{0}\right), \quad D=\operatorname{diag}\left(D_{1}, D_{2}\right), \\
& D_{1}=V_{01}, \quad D_{2}=\left\{\begin{array}{l}
\operatorname{diag}\left(\lambda^{\gamma / 2} \xi^{-r}, \lambda^{-\gamma / 2} \xi^{r}\right) \\
\varphi^{-\frac{1}{4}} I_{2}
\end{array} \quad \text { as } \quad x>b_{\lambda},\right. \\
& r=\frac{1}{2}\left(\sqrt{(\beta-1)^{2}+2 C}+1\right), \\
& \widetilde{V}_{0}=P^{-1} V_{0} D^{-1}, \quad \widetilde{V}=b_{\lambda}^{-1} V D^{-1} .
\end{aligned}
$$

Then $\widetilde{V}_{0}=\operatorname{diag}\left(I_{2}, \widetilde{V_{02}}\right)$, at that, see $\left[22\right.$, Sect. 7.13], as $x \leqslant M^{-1} \lambda^{1 /(\beta-2)},(M \gg 1)$, we have

$$
\begin{aligned}
\widetilde{V_{02}}= & \sqrt{\frac{\beta-2}{\pi}}\left(\begin{array}{cc}
\cos \Phi_{1} & \sin \Phi_{1} \\
\sin \Phi_{1} & -\cos \Phi_{1}
\end{array}\right) \\
& \cdot\left[I_{2}+\frac{1}{2}\left(\gamma^{2}-\frac{1}{4}\right)(\sqrt{\lambda})^{-1}\left(\begin{array}{cc}
0 & 1 \\
-1 & 0
\end{array}\right)+O\left(\varphi^{-\frac{1}{2}} \frac{\nu^{\prime}}{\nu}+\frac{1}{\lambda Q_{2}^{2}}\right)\right], \\
\Phi_{1}= & Q_{2}-(2 \gamma+1) \pi / 4 .
\end{aligned}
$$

Moreover, for sufficiently large $M>0, \Lambda_{0}>0$ there exist positive constants $C_{1}, C_{2}$ such that

$$
C_{1}<\mid \widetilde{V_{02}}\left(x, \lambda \mid<C_{2} \quad \text { for all } \quad x \geqslant M \lambda^{\frac{1}{\beta-2}}, \quad \lambda \geqslant \Lambda_{0} .\right.
$$

3.3. Integral equation. We have,

$$
V=V_{0}+\int_{\Gamma(x)} V_{0}(x, \lambda) V_{0}(t, \lambda) Z(t, \lambda) V(t, \lambda) d t
$$

where $Z=Z_{1}-Z_{2}$, see (31) and (33). We multiply left and right hand side of this equation by $P^{-1}$ and $D^{-1}$, respectively (cf. $(36)$ ), and we obtain the equation for $\widetilde{V}$

$$
\widetilde{V}=\widetilde{V}_{0}+A(\lambda) \widetilde{V}
$$

where the operator $A(\lambda)$ acts by the formula:

$$
\begin{aligned}
& (A(\lambda) \widetilde{V})(x, \lambda)=\widetilde{V}_{0}(x, \lambda) \int_{\Gamma(x)} A(x, t, \lambda) \widetilde{V}(t, \lambda) D(t, \lambda) D^{-1}(x, \lambda) d t=: \widetilde{V}_{0}(x, \lambda) A_{1}(\lambda) \widetilde{V}, \\
& A(x, t, \lambda)=D(x, \lambda) D^{-1}(t, \lambda)\left(\widetilde{V}_{0}^{-1} P^{-1} Z P\right)(t, \lambda) .
\end{aligned}
$$

By $\Gamma(x)=\left(\left(\gamma_{i j}, x\right)\right)$ we denote a matrix with entries $\left(\gamma_{i j}, x\right)$ being the intervals over which we integrate the element standing in $i$ th row and $j$ th column of the matrix integrand. We choose them as follows: $\gamma_{i j}=+\infty$ as $(i, j)=(3,2),(4,2),(4,3)$ as $\gamma_{i j}=0$ for other $(i, j)$. It follows from definition (34) - (35) of the matrix $D$ that under such choice all exponential factors in (40) are bounded. Then the norm of the operator $A(\lambda)$ in the space $Z$ satisfies the estimate

$$
\|A(\lambda)\|=O(I(\lambda)), \quad I(\lambda)=\int_{0}^{\infty}\|G(t, \lambda)\| d t,
$$




$$
G(t, \lambda)=P^{-1}(t, \lambda) Z(t, \lambda) P(t, \lambda) .
$$

Straightforward calculations show that

$$
G(t, \lambda)=\operatorname{diag}\left(0, G_{0}\right)+O(g)
$$

where

$$
\begin{aligned}
& G_{0}=\left(\begin{array}{cc}
0 & g_{1} \\
g_{2} & 0
\end{array}\right), \quad g=\nu_{1}^{-\frac{1}{2}}\left(\left(\frac{\nu_{1}^{\prime}}{\nu_{1}}\right)^{2}+\frac{\nu_{1}^{\prime \prime}}{\nu_{1}}\right), \\
& g_{1}=\frac{1}{8} \frac{p_{0}}{\sqrt{D}}\left(\frac{D^{\prime}}{D} \frac{\nu_{1}^{\prime}}{\sqrt{D}}+8 \chi p\right), \\
& g_{2}=\frac{1}{4 p_{0}}\left(-\frac{1}{4}\left(\frac{\nu^{\prime}}{\nu}\right)^{2}+\frac{\nu^{\prime \prime}}{\nu}-\frac{\beta(3 \beta-4)+8 c}{(\beta-2)^{2}} \lambda Q_{2}^{-2} \nu^{-1}-\frac{4 q}{\nu_{1}}\right) .
\end{aligned}
$$

Lemma 1. Let the functions $p$ and $q$ satisfy Conditions 1)-3), and in the case $\beta=\alpha+2$, Condition 4) is satisfied as well. Then

$$
\begin{aligned}
& I(\lambda)=O\left(\lambda^{-m}\right), \lambda \rightarrow+\infty, \\
& m= \begin{cases}\min \left\{\frac{1}{4}, \frac{\beta-\alpha-2}{\beta-2}, \frac{\varepsilon}{\beta-2}\right\} & \text { as } \beta>\alpha+2, \\
\min \left\{\frac{1}{4}, \frac{\sigma}{\beta-2}, \frac{\varepsilon}{\beta-2}\right\} & \text { as } \beta=\alpha+2,\end{cases}
\end{aligned}
$$

where $\sigma$ and $\varepsilon$ are positive constants from Conditions 3) and 4).

Proof. By relations 41) and (42) we have

$$
I(\lambda)=O\left(\int_{0}^{\infty}\left(g+\left|g_{1}\right|+\left|g_{2}\right|\right) d t\right) .
$$

Simple estimates based on inequalities (14), (16) and Condition 2) show that

$$
\int_{0}^{\infty}\left(g+\left|g_{1}\right|\right) d t=O\left(\lambda^{-\frac{1}{4}-\frac{1}{2 \beta}}\right), \quad \lambda \rightarrow+\infty .
$$

In order to estimate the integral $I_{1}(\lambda)=\int_{0}^{\infty}\left|g_{2}\right| d t$, we split it into two integrals

$$
I_{1}=\left(\int_{0}^{c_{\lambda}}+\int_{c_{\lambda}}^{\infty}\right)\left|g_{2}\right| d t=I_{11}+I_{12}, \quad c_{\lambda}=\lambda^{1 / 2 \beta},
$$

and we observe that Conditions 3), 4) imply:

$$
\begin{aligned}
& g_{2}=O\left(x^{-2}\left(x^{-\delta}+\lambda x^{-2 \beta}\right)\right), \quad c_{\lambda}^{-1} x \rightarrow \infty, \\
& \delta= \begin{cases}\min \{\varepsilon, \beta-\alpha-2\} & \text { as } \beta>\alpha+2, \\
\min \{\varepsilon, \sigma\} & \text { as } \beta=\alpha+2 .\end{cases}
\end{aligned}
$$

This easily yields 45).

Three numbers determining $m$ in (45) are of different nature. The first number depends on the smoothness of the function $p$ in the vicinity of zero. The second number, $\frac{\beta-\alpha-2}{\beta-2}$ or $\frac{\sigma}{\beta-2}$, is subject to the choice of reference solutions and in the sense, it characterizes the precision of the method. The latter number is determined by the relative smallness of the function $R(x)=p(x)-x^{\beta}$ in the vicinity of infinity. In the limiting case, we assume that the function 
$R(x)$ is compactly supported, the number $\varepsilon$ disappears in 46$)$. If we additionally assume that the function $V=q-x^{\alpha}$ in Condition 4) is compactly supported, then as $\beta=\alpha+2$, we have

$$
g_{2}=O\left(\lambda x^{-2-2 \beta}\right), \quad c_{\lambda}^{-1} x \rightarrow \infty .
$$

Thus, the following lemma holds.

Lemma 2. Let the functions $p$ and $q$ be of form (18). Then

$$
\begin{aligned}
& I(\lambda)=O\left(\lambda^{-m}\right), \quad \lambda \rightarrow \infty, \\
& m=\left\{\begin{array}{cl}
\min \left\{\frac{1}{4}+\frac{1}{2 \beta}, \frac{\beta-\alpha-2}{\beta-2}\right\} & \text { as } \beta>\alpha+2, \\
\frac{1}{4}+\frac{1}{2 \beta} & \text { as } \beta=\alpha+2 .
\end{array}\right.
\end{aligned}
$$

3.4. Completion of proof of Theorem 1. It follows from Lemma 1 that as $\lambda \rightarrow \infty$,

$$
Y(x, \lambda)=T P\left(I_{4}+o(1)\right) \widetilde{V}_{0}\left(I_{4}+o(1)\right) D(x, \lambda)
$$

uniformly in $x \geqslant 0$. Hence, the deficiency indices of the minimal operator $T_{0}$ are equal $(2,2)$. Hence, the operator $T$ is self-adjoin and the equation for the eigenvalues is of the form:

$$
\operatorname{det}\left[C_{2} \widetilde{V}(0, \lambda)\left(E_{4}+A_{1}(\lambda) \widetilde{V}_{0}(0, \lambda)+O\left(I^{2}(\lambda)\right) C_{1}^{T}\right]=0,\right.
$$

where

$$
C_{1}=\left(\begin{array}{cccc}
0 & 1 & 0 & 0 \\
0 & 0 & 1 & 0
\end{array}\right), \quad C_{2}=\left(\begin{array}{cccc}
1 & 1 & 0 & 0 \\
0 & 0 & 1 & 0
\end{array}\right) .
$$

It is easy to check that $\left[A_{1}(\lambda) \widetilde{V}_{0} C_{1}^{T}\right](0, \lambda)$ is of the form

$$
\left(A_{1}(\lambda) \widetilde{V}_{0} C_{1}^{T}\right)(0, \lambda)=\left(\begin{array}{cc}
\alpha_{11} & \alpha_{12} \\
0 & 0 \\
\alpha_{31} & 0 \\
\alpha_{41} & \alpha_{42}
\end{array}\right)
$$

and

$$
\alpha_{42}=\frac{\pi}{\beta-2} \int_{0}^{\infty}\left(g_{1} \omega_{12} \omega_{21}-g_{2} \omega_{11}^{2}\right) f(t, \lambda) d t+O(|\beta(\lambda)|),
$$

where $\omega_{i j}$ denote the entries of the matrix $\widetilde{V_{02}}$,

$$
\begin{aligned}
& f(t, \lambda)= \begin{cases}1 & \text { as } t \leqslant b_{\lambda}, \\
\lambda^{\gamma} \xi^{-(\beta-1)}(t, \lambda) & \text { as } t>b_{\lambda},\end{cases} \\
& \beta(\lambda)=\lambda^{-\frac{1}{2}} \int_{0}^{\infty}\left(\frac{q_{1}^{\prime} p_{1} p_{1}^{\prime}}{\left(p_{1}^{2}+\lambda\right)^{\frac{7}{4}}}+\frac{q_{1}^{\prime \prime}}{\left(p_{1}^{2}+\lambda\right)^{\frac{3}{4}}}\right) d t,
\end{aligned}
$$

and all other non-zero entries in (48) obey the estimate

$$
\alpha_{i j}=O\left(\lambda^{-1 / 4} \int_{0}^{x_{0}+1}|\chi p| \exp \left(-\delta_{0} Q_{1}\right) d t\right)+O\left(\lambda^{-\frac{3}{4}}\right)+O\left(Q_{2}^{-2}(0, \lambda)\right), \quad(i, j) \neq(4,2) .
$$

But

$$
\beta(\lambda)=O\left(\lambda^{-\frac{3}{4}-\frac{\beta-\alpha-1}{2 \beta}}\right),
$$

therefore, equation (47) can be written as

$$
\omega_{11}(0, \lambda)+\alpha_{42}(\lambda) \omega_{12}(0, \lambda)+O\left(I^{2}(\lambda)+Q_{2}^{-2}(0, \lambda)\right)=0 .
$$

Let

$$
K(\lambda)=\frac{\beta(3 \beta-4)+8 c}{8(\beta-2)^{2}} Q_{2}^{-1}(0, \lambda)+\frac{\pi}{\beta-2} \int_{0}^{\infty}\left(g_{1} \omega_{21} \omega_{12}-\omega_{11}^{2} g_{2}\right) f(t, \lambda) d t
$$


where $C, f(t, \lambda), g_{1}, g_{2}$ are defined respectively by (21), (49), (43), (44). Replying $\omega_{11}$ and $\omega_{12}$ by their asymptotics in (52) in accordance with (37), we obtain equation (19). The proof is complete.

\section{Proof of Theorem 2}

4.1. Quantization parameter. It follows from equation 19 that

$$
\Phi\left(\lambda_{k}\right)=\nu_{k} \pi+o(1), k \rightarrow \infty,
$$

where $\nu_{k}=\nu_{k}(\alpha, \beta)$ is a positive integer number, a quantization parameter. Let us show that $\nu_{k}(\alpha, \beta)=k$.

Let $\lambda_{k}(\beta)$ be a $k$ th eigenvalue of the operator $T$ as $p=x^{\beta}, q=0$ and let $\left\{\mu_{k}\right\}_{1}^{\infty}$ be the spectrum of the problem

$$
\begin{aligned}
& y^{(4)}=\lambda y, \quad 0 \leqslant x \leqslant 1, \\
& y(0)=y^{\prime \prime}(0)=0, \quad y(1)=y^{\prime}(1)=0 .
\end{aligned}
$$

Lemma 3. For each fixed $k$

$$
\lambda_{k}(\beta) \rightarrow \mu_{k}, \quad \beta \rightarrow+\infty .
$$

Proof. It follows from Lemma 2 of work [12] that the quadratic form of the operator $T$ reads as

$$
\begin{aligned}
& l[y]=\int_{0}^{\infty}\left(\left|y^{\prime \prime}\right|^{2}+2 x^{\beta}\left|y^{\prime}\right|^{2}\right) d x, \\
& D(l)=\left\{y \in L^{2}(0, \infty): y, y^{\prime} \in A C[0,+\infty), y^{\prime \prime} \in L^{2}(0, \infty), y(0)=0\right\} .
\end{aligned}
$$

We denote by $y_{k}(\beta)=y_{k}(\beta, x)$ and $z_{k}=z_{k}(x)$ the $k$ th normalized eigenfunction of the operator $T$ and of problem (54)-(55), respectively. Then if we continue $z_{k}$ by zero on $[1, \infty)$, then $z_{k} \in D(l)$ and $l\left[z_{k}\right]=\mu_{k}+\varepsilon_{k}$, where $\varepsilon_{k}=\int_{0}^{1} 2 x^{\beta}\left|f_{k}^{\prime}\right|^{2} d x$. By the minimax principle $\mid 13$, Ch. XIII, Thm. XIII.3] we conclude that

$$
\lambda_{k}(\beta) \leqslant \mu_{k}+\varepsilon_{k} \quad(k=1,2, \ldots),
$$

and for each fixed $k$ we have $\varepsilon_{k} \rightarrow+0$ as $\beta \rightarrow+\infty$. Then employing the inequalities

$$
\int_{1}^{\infty} x^{\beta}\left|y_{k}^{\prime}(\beta, x)\right|^{2} d x \leqslant \mu_{k}+\varepsilon_{k}, \quad \int_{1}^{\infty}\left|y_{k}^{\prime \prime}(\beta, x)\right|^{2} d x \leqslant \mu_{k}+\varepsilon_{k},
$$

by simple calculations we get that

$$
y_{k}(1)=o(1), \quad y_{k}^{\prime}(1)=o(1), \quad \beta \rightarrow+\infty .
$$

for each fixed $k$. But

$$
\int_{0}^{1}\left|y_{k}^{\prime \prime}(\beta, x)\right|^{2} d x<\lambda_{k}(\beta)
$$

and hence, taking into consideration (57), we conclude tha fo each $\varepsilon>0$ and for each $k \in \mathbb{N}$ there exists a positive number $B(k, \varepsilon)$ such that for each $\beta>B(k, \varepsilon)$ there exists a function $v_{k}$ in the domain of quadratic form $(54)-55$, for which

$$
\int_{0}^{1}\left|v_{k}^{\prime \prime}\right|^{2} d x<\lambda_{k}(\beta)+\varepsilon
$$

By latter inequality and the minimax principle we obtain:

$$
\mu_{k} \leqslant \lambda_{k}(\beta)+\varepsilon, \quad \beta>B(k, \varepsilon) .
$$

Together with inequality 56 this complete the proof. 
Lemma 4. For each $\beta>2$

$$
\Phi\left(\lambda_{k}(\beta)\right)=k \pi+o(1), \quad k \rightarrow \infty .
$$

Proof. The equation for the eigenvalues of problem (54)- 55) is of the form

$$
\sin \left(\mu^{\frac{1}{4}}-\frac{\pi}{4}\right)+\exp \left(-2 \mu^{\frac{1}{4}}\right) \cos \left(\mu^{\frac{1}{4}}-\frac{\pi}{4}\right)=0,
$$

and applying Rouché theorem, we obtain

$$
\mu_{k}^{\frac{1}{4}}=\pi\left(k+\frac{1}{4}\right)+o(1), \quad k \rightarrow+\infty .
$$

But as $\beta \rightarrow \infty($ see $(20))$,

$$
\Phi(\lambda)=\lambda^{\frac{1}{4}}-\frac{\pi}{4}+o(1),
$$

and in (53) we have $\nu_{k}(\beta)=k$ for sufficiently large $\beta$. According Theorem 1 in [12], the function $\lambda_{k}(\cdot)$ is continuous on $(2, \infty)$ and therefore, the function $\nu_{k}(\cdot)$ is also continuous on $(2, \infty)$, so that $\nu_{k}(\beta)=k$ as $\beta>2$.

Lemma 5. Let $\lambda_{k}(\alpha, \beta)_{1}^{\infty}$ be the eigenvalues of the operator $T$ with $q(x)=x^{\alpha}, p=x^{\beta}$. Then

$$
\Phi\left(\lambda_{k}(\alpha, \beta)\right)=k \pi+o(1), \quad k \rightarrow \infty .
$$

Proof. Since $\lambda_{k}(\alpha, \beta)$ is continuous on $\Omega=\{\beta \geqslant 2+\alpha, \alpha>-1\} \cup\{\beta>2\}$, see Theorem 1 in [12]) and the term $o(1)$ in (53) is small uniformly in each compact set $K$ in $\Omega$, then $\nu_{k}(\alpha, \beta)$ is continuous on $\Omega$. But $\nu_{k}(0, \beta)=k$, cf. Lemma 15 , therefore, $\nu_{k}(\alpha, \beta)=k$ in $\Omega$.

4.2. Completion of proof of Theorem 2. We have

$$
Q_{2}(0, \lambda)=\lambda^{\frac{1}{4}+\frac{1}{2 \beta}} \int_{0}^{\infty}\left(t^{\beta}+\sqrt{t^{2 \beta}+1}\right)^{-\frac{1}{2}} d t=C_{0} \lambda^{\frac{1}{4}+\frac{1}{2 \beta}}
$$

and the problem is hence reduced to studying the asymptotics of $K(\lambda)$. Replacing $\omega_{11}, \omega_{12}$, $\omega_{21}$ in integral $(52)$ by their asymptotics according $(37)$, changing the variable $t \longmapsto \lambda^{\frac{1}{2 \beta}} \tau$, we obtain supposing $\chi=0$ in 43 :

$$
\begin{aligned}
& K(\lambda)=-C_{1} \lambda^{-\frac{1}{4}}+C_{2} \lambda^{-\frac{1}{4}-\frac{1}{2 \beta}}+k(\lambda)+O\left(\lambda^{-\frac{1}{2}-\frac{1}{2 \beta}}\right), \\
& k(\lambda)=\frac{\pi}{\beta-2} \int_{0}^{\infty} p_{0}^{-1} q \nu^{-1} \omega_{11}^{2} f(t, \lambda) d t .
\end{aligned}
$$

By the definition of $\widetilde{V}_{0}(x, \lambda)$ (see $(36)$ ) we have

$$
k(\lambda)=\frac{\pi}{2} \int_{0}^{\infty} \lambda^{-\frac{1}{2}} q \nu^{-\frac{1}{2}} Q(t, \lambda) J_{\gamma}^{2}(\sqrt{\lambda} Q) d t,
$$

where

$$
Q=\int_{t}^{\infty} \nu^{-\frac{1}{2}} d t
$$

Let $\beta<2 \alpha+2$. Then splitting the integral $k(\lambda)$ into the sum

$$
k(\lambda)=\frac{\pi}{2}\left[\int_{0}^{c_{\lambda}}+\int_{c_{\lambda}}^{\infty}\right] \lambda^{-\frac{1}{2}} q \nu^{-\frac{1}{2}} Q J_{\gamma}^{2}(\sqrt{\lambda} Q) d t,
$$

where

$$
c_{\lambda}=b_{\lambda} \lambda^{-\varepsilon}, \quad 0<\varepsilon<\frac{1}{\beta-2}-\frac{1}{2 \beta}
$$


and replacing $J_{\gamma}(\sqrt{\lambda} Q)$ by its asymptotics in the first integral and replacing $q$ in the second integral by the expression $\left(\frac{\sqrt{2}}{\beta-2} Q\right)^{-\frac{2 \alpha}{\beta-2}}$ thanks to Conditions 3) and 4), we obtain

$$
k(\lambda)=C_{3} \lambda^{-\frac{\beta-\alpha-2}{\beta-2}}+O\left(\lambda^{-\frac{1}{2}-\frac{\beta-\alpha-2}{\beta-2}}\right) .
$$

Substituting now $K$ into equation 19 and resolving it with respect to $\lambda$ taking into consideration Lemma 5, we obtain (25). The proof is complete.

Remark 2. The case $\beta \geqslant 2 \alpha+2$ differs from the above considered one only by the asymptotics of the integral $k(\lambda)$. It is easy to see that as $\beta>2 \alpha+2$, we have

$$
k(\lambda) \sim \operatorname{const} \lambda^{-\frac{1}{2}-\frac{\beta-2-2 \alpha}{4 \beta}},
$$

and the term $C_{3} m_{k}^{-\frac{1}{2}+\frac{2 \alpha \beta}{\beta^{2}-4}}$ in (25) should be replaced by the term of form const $\cdot m_{k}^{-\frac{1}{2}+\frac{\alpha}{\beta+2}}$. As $\beta=2 \alpha+2$, we have $k(\lambda)=$ const $\cdot \lambda^{-\frac{1}{2}} \ln \lambda$ and this gives rise to the term of form const $\cdot m_{k}^{-\frac{2}{\beta-2}} \ln m_{k}$ in 25 .

Theorem 3. Let the functions $p$ and $q$ be of form (18) and $\beta=\alpha+2$. Then

$$
\lambda_{k}=m_{k}^{\frac{4 \beta}{\beta+2}}+\frac{4 \beta}{\beta+2} C_{0}^{-1}\left\{C_{1} m_{k}^{\frac{5(\beta-4)}{2(\beta+2)}}-C_{2} m_{k}^{\frac{2(\beta-2)}{\beta+2}}\right\}+O\left(k^{-m}\right),
$$

where $m_{k}=C_{0}^{-1} \pi\left(k-\frac{1}{4}+\frac{\sqrt{(\beta-1)^{2}+2}}{2(\beta-2)}\right)$, the constants $C_{0}, C_{1}$ are defined in the same way as in the case $\beta>2+\alpha$ :

$$
\begin{aligned}
C_{2}= & \frac{\beta}{8} \int_{0}^{\infty}\left[\widetilde{\nu}^{\frac{1}{2}} \widetilde{D}^{-\frac{1}{2}} t^{\beta-2}\left(-\frac{2 \beta}{\widetilde{D}}+\frac{\beta}{\widetilde{D}^{2}}-\frac{3 \beta t^{\beta}}{\sqrt{\widetilde{D}}}+1\right)\right. \\
& \left.+\frac{3(\beta-4) \beta+8}{\beta(\beta-2)^{2}} \widetilde{Q}^{-2} \widetilde{\nu}^{-\frac{1}{2}}-8 t^{\beta-2} \widetilde{\nu}^{-1}\right] d t-\frac{3(\beta-4) \beta+8}{(\beta-2)^{2}} C_{0}^{-1}, \\
m= & \min \left\{\frac{1}{2}+\frac{1}{\beta}, \frac{3}{4}, \frac{2(\beta-2-\alpha)}{\beta-2}\right\}-\frac{3 \beta-2}{\beta+2} .
\end{aligned}
$$

Proof. Arguing as in the proof of the previous theorem, we get

$$
K(\lambda)=-C_{1} \lambda^{-\frac{1}{4}}+C_{2} \lambda^{-\frac{1}{4}-\frac{1}{2 \beta}}+O\left(\lambda^{-1 / 2-1 / \beta}\right),
$$

and this implies the statement of the theorem.

\section{On DENSITY OF EIGENVALUES AND SINGULAR VALUES OF NON-SELF-ADJOINT ANHARMONIC OSCILLATOR}

Let $H(\alpha, \theta)$ be an operator acting in $L^{2}(0,+\infty)$ by the rule

$$
\begin{aligned}
& D(H(\alpha, \theta))=\left\{y \in L^{2}(0,+\infty): y, y^{\prime} \in A C[0,+\infty),\right. \\
& \left.-y^{\prime \prime}+e^{i \theta} x^{\alpha} y \in L^{2}(0,+\infty), y(0)=0\right\}, \\
& H(\alpha, \theta) y=-y^{\prime \prime}+e^{i \theta} x^{\alpha} y .
\end{aligned}
$$

Here $\theta \in(-\pi, \pi), \alpha \in(0,+\infty)$ are constants, $A C[0,+\infty)$ is the set of the functions absolutely continuous on each segment $[0, a], a>0$. The operator $H(\alpha, \theta)$ is called a non-self-adjoint anharmonic oscillator [23]. 
The operator $H(\alpha, \theta)$ was studied by many authors, see [20], [24], [25], [23], [26] and the references therein. It is known, cf. [24], [20], that for each $|\theta|<\pi$ the spectrum of $H(\alpha, \theta)$ is discrete, all eigenvalues are simple (of algebraic multiplicity 1 ), are located on the ray $\arg z=$ $\frac{2 \theta}{2+\alpha}$ :

$$
\begin{aligned}
& \lambda_{n}(\alpha, \theta)=\lambda_{n}(\alpha, 0) e^{\frac{2 \theta i}{2+\alpha}}, \\
& \lambda_{n}(\alpha, 0) \sim\left(\frac{\pi}{\int_{0}^{1} \sqrt{1-t^{\alpha}} d t}\right)^{\frac{2 \alpha}{2+\alpha}} n^{\frac{2 \alpha}{2+\alpha}}, \quad n \rightarrow \infty .
\end{aligned}
$$

As it was shown in [23],

$$
\left\|\left(H(\alpha, \theta)-r e^{i \beta}\right)^{-1}\right\| \rightarrow \infty, \quad r \rightarrow+\infty,
$$

uniformly in $\beta \in\left[\delta, \frac{2 \theta}{2+\alpha}-\delta\right] \cup\left[\frac{2 \theta}{2+\alpha}+\delta, \theta-\delta\right], \delta>0$. Therefore, for arbitrary small $\delta>0$ there exists $R_{\delta}>0$ such that $\varepsilon$-pseudospectrum of the operator $H(\alpha, \theta)$

$$
\sigma_{\varepsilon}(H(\alpha, \theta))=\sigma(H(\alpha, \theta)) \cup\left\{z \in \mathbb{C} \backslash \sigma(H(\alpha, \theta)):\left\|(H(\alpha, \theta)-z)^{-1}\right\| \geqslant \varepsilon^{-1}\right\}
$$

contains the sectors

$$
\left\{r e^{i \beta}: r>R_{\delta}, \delta \leqslant \beta \leqslant \frac{2 \theta}{2+\alpha}-\delta\right\} \quad \text { and } \quad\left\{r e^{i \beta}: r>R_{\delta}, \frac{2 \theta}{2+\alpha}+\delta \leqslant \beta \leqslant \theta-\delta\right\} .
$$

According a known formula [27, we have

$$
\sigma_{\varepsilon}(T)=\overline{\left\{\bigcup_{\|V\| \leqslant \varepsilon} \sigma(T+V)\right\}} .
$$

This means that the operator $H(\alpha, \theta)$ is spectrally unstable: its spectrum can change essentially under rather small perturbations [28]. While studying the spectral properties of the perturbations of such operators by traditional for self-adjoint case methods based on Tauberian theorems (see $[9]$ ), an estimate for the density of so-called singular values is important; these numbers are the eigenvalues of the absolute value of the operator: $|H(\alpha, \theta)|=\sqrt{H(\alpha, \theta)^{*} H(\alpha, \theta)}$. It is easy to confirm that $M:=H(\alpha, \theta)^{*} H(\alpha, \theta)$ is a self-adjoint operator in $L^{2}(0,+\infty)$ generated by the differential expression $\bar{l}(l y)$ and the boundary conditions $y(0)=y^{\prime \prime}(0)=0$, where $l y=-y^{\prime \prime}+q y$, $\bar{l} y=-y^{\prime \prime}+\bar{q} y$. By help of the standard change $Y=\left(y, l y, y^{\prime},(l y)^{\prime}\right)^{t}$, the equation $M y=s y$ is reduced to the spectral problem

$$
\begin{aligned}
& Y^{\prime}=A Y, \\
& Y_{1}(0)=Y_{3}(0)=0,
\end{aligned}
$$

where

$$
A=\left(\begin{array}{cccc}
0 & 0 & 1 & 0 \\
0 & 0 & 0 & 1 \\
q & -1 & 0 & 0 \\
-s & \bar{q} & 0 & 0
\end{array}\right)
$$

The eigenvalues of the matrix $A$ are $\pm \sqrt{\cos \theta r \pm \sqrt{s-\sin ^{2} \theta r^{2}}}$, and hence, for large $s>0$, equation 62 possesses two turning points, at which one or two pairs of the eigenvalues coincide. These points generate additional troubles while studying the asymptotics of solutions to equation (62). Nevertheless, these troubles are pure technical and they can be overcome as above, by means of reference equations method. In contrast to equation (7), both turning points of equation 62 are finite and this is why reference solutions corresponding to these 
points are to be expressed in terms of the Airy functions. Not dwelling on details, we only note that thanks to coercive estimate

$$
(M y, y) \geqslant(1-\delta)\left(\left\|y^{\prime \prime}\right\|^{2}+\|r y\|^{2}\right)-C_{\delta}\|y\|^{2},
$$

where $0<\delta=\delta(\theta)<1, C_{\delta}>0, r=x^{\alpha}$, one can obtain a needed estimate:

$$
\liminf _{\lambda \rightarrow+\infty} \frac{N(H(\alpha, \theta), \lambda)}{N(|H(\alpha, \theta)|, \lambda)}>0
$$

see Theorem 1 in $[9]$. We stress once again that calculating at least the leading term in the asymptotics for the spectrum of the operator $M$ as in Sections 4, 5 or employing Tauberian technique as in work [17], one can show that

$$
N(|H(\alpha, \theta)|, \lambda) \sim \text { const } \cdot \lambda^{\frac{2 \alpha}{2+\alpha}}, \quad \lambda \rightarrow+\infty .
$$

In view of this we mention work [29], in which formula (63) was established for an arbitrary dissipative operator $A$ in the Shatten-von Neumann class $\mathfrak{S}_{p}$ as $p \leqslant \frac{\pi}{2 \theta_{A}}$, where $\theta_{A}$ is the opening of the angle

$$
\operatorname{Num}(A)=\{(A f, f): f \in D(A),\|f\|=1\} .
$$

In our case the latter assumption is absent.

\section{BIBLIOGRAPHY}

1. V.V. Buldygin, O.I. Klesov, J.G. Steinebach. Properties of a subclass of avakumovic functions and their generalized inverses // Ukr. Math. Jour. 54:2, 179-206 (2002).

2. V.V. Buldygin, O.I. Klesov, J.G. Steinebach. On some extensions of Karamata's theory and their applications // Publ. Inst. Math. Nouv. Ser. 80(94), 59-96 (2006).

3. J. Karamata. Sur un mode de croissance régulière des fonctions // Mathematica. 4, 33-53 (1930).

4. E. Seneta. Regularly varying functions. Lect. Notes Math. 508. Springer-Verlag, Berlin (1976).

5. N.H. Bingham, C.M. Goldie, J.L. Teugels. Regular variation. Encyclopedia of mathematics and its applications. 27. Cambridge Univ. Press, Cambridge (1987).

6. T. Carleman. Über die asymptotische Verteilung der Eigenverte partieller Differentialgleichungen // Ber. Sachs. Acad. Wiss. Leipzig. 88, 119-132 (1936).

7. A.G. Kostyuchenko, I.S. Sargsyan. Distribution of eigenvalues. Selfadjoint ordinary differential operators. Nauka, Moscow (1979). (in Russian).

8. A.A. Shkalikov. Theorems of Tauberian type on the distribution of zeros of holomorphic functions // Matem. Sborn. 123(165):3, 317-347 (1984). [Math. USSR-Sb. 51:2, 315-344 (1985)].

9. Kh.K. Ishkin. Conditions of spectrum localization for operators not close to self-adjoint operators // Dokl. RAN. 479:5, 1-4 (2018). [Dokl. Math. 97:2, 170-173 (2018).]

10. M.A. Naimark. Linear differential operators. Fizmatlit, Moscow (1969). [Frederick Ungar Publishing Co., New York. Part I. (1967); Part II. (1968).]

11. M.V. Fedoryuk. Asymptotic analysis: linear ordinary differential equations. Nauka, Moscow (1983). [Springer-Verlag, Berlin (1993).]

12. Kh.K. Ishkin. On continuity of the spectrum of a singular quasi-differential operator with respect to a parameter // Eurasian Math. J. 2:3, 67-81 (2011).

13. M. Reed, B. Simon. Methods of modern mathematical physics. 4. Academic Press, New York (1978).

14. Ja.T. Sultanaev. Asymptotic behavior of the spectrum of singular differential operators in the indefinite case // Vestnik Mosc. Univ. Ser. Matem. Mekh. 3, 21-30 (1975). [Mosc. Univ. Math. Bull. 30:3-4, 15-22 (1975).]

15. Kh.K. Ishkin. Asymptotic behavior of the spectrum and the regularized trace of higher-order singular differential operators // Differ. Uravn. 31:10, 1658-1668 (1995). [Differ. Equat. 31:10, 16221632 (1995).]

16. G.V. Rozenblum, M.Z. Solomyak, M.A. Shubin. Spectral theory of differential operators // Itogi Nauki i Tekhniki. Ser. Sovrem. Probl. Mat. Fund. Napr. Partial differential equations-7. 64, 5-242 (1989). (in Russian). 
17. Ya.T. Sultanaev. A two-sided Tauberian theorem for ratios // Izv. VUZ. Matem. 140:1, 103-112 (1974). [Soviet Math. 18:1, 84-91 (1974).]

18. R.E. Langer. The asymptotic solutions of ordinary linear differential equations of the second order with special reference to the Stokes' phenomenon // Bull. Amer. Math. Soc. 40:8, 545-582 (1934).

19. M.A. Evgrafov, M.V. Fedoryuk. Asymptotic behaviour as $\lambda \rightarrow \infty$ of the solution of the equation $w^{\prime \prime}(z)-p(z, \lambda) w(z)=0$ in the complex $z$-plane // Uspekhi Matem. Nauk. 21:1, 3-50 (1966). [Russ. Math. Surv. 21:1, 1-48 (1966).]

20. Kh.K. Ishkin. On the spectral instability of the Sturm-Liouville operator with a complex potential. // Diff. Uravn. 45:4, 480-495 (2009). [Diff. Eqs. 45:4, 494-509 (2009).]

21. Kh.K. Ishkin, Kh.Kh. Murtazin. Asymptotics for the eigenvalues of a fourth order differential operator in a "degenerate" case // Ufimskij Matem. Zhur. 8:3, 82-98 (2016). [Ufa Math. J. 8:3, 79-94 (2016).]

22. H. Bateman, A. Erdélyi. Higher transcendental functions. V. 2. MC Graw-Hill Book Co., New York (1974).

23. E. B. Davies. Wild spectral behaviour on anharmonic oscillators // Bull. London Math. Soc. 32:4, 432-438 (2000).

24. V. B. Lidskii. Conditions for completeness of a system of root subspaces for non-selfadjoint operators with discrete spectrum // Trudy MMO. 8, 83-120 (1959). (in Russian).

25. V. B. Lidskii. A non-selfadjoint operator of Sturm-Liouville type with a discrete spectrum // Trudy MMO. 9, 45-79 (1960). (in Russian).

26. E.B. Davies. Pseudo-spectra, the harmonic oscillator and complex resonances // Proc. Royal Soc. London. 455:1982, 585-599 (1999).

27. S. Roch, B. Silberman. $C^{*}$-algebra techniques in numerical analysis // J. Oper. Theory. 35:2, 221-280 (1996).

28. Kh.K. Ishkin. A localization criterion for the eigenvalues of a spectrally unstable operator // Dokl. RAN. 429:3, 301-304 (2009). [Dokl. Math. 80:3, 829-832 (2009).]

29. K.Kh. Boimatov. Asymptotic behavior of eigenvalues of non-self-adjoint operators // Funkts. Anal. Pril. 11:4, 74-75 (1977). [Funct. Anal. Appl. 11:4, 305-306 (1977).]

Lyajsan Gabdulkhaevna Valiullina,

Bashkir State University,

Zaki Validi str. 32,

450074, Ufa, Russia

E-mail: 1.matem2012@yandex.ru

Khabir Kabirovich Ishkin,

Bashkir State University,

Zaki Validi str. 32,

450074, Ufa, Russia

E-mail: Ishkin62@mail.ru

Rustem Ildarovich Mardanov,

Bashkir State University,

Zaki Validi str. 32,

450074, Ufa, Russia

E-mail: rsmar1v@gmail.com 